\title{
Editorial: Digitalisation and Artificial Intelligence in European Healthcare
}

\section{Introduction}

A few months ago I was appointed editor of the European Journal of Health Law, a publication to which I hope to contribute in the best possible way during the coming years. Health law is indeed a crucial area of the law, where the national meets the international, experts on law meet healthcare personnel, and policymaking and legislation often bear directly on large portions of the population. We live in a time with many challenges - medical, ethical, and social - in connection with healthcare and people's health all over Europe and the rest of the world. Add to this the fact that health law is an area in rapid development, due to digitalisation and automated decision-making in many parts of the production and distribution of healthcare. These developments offer great opportunities, of course, but they also pose serious challenges. Legal research must respond to these challenges, and solely national fora are insufficient for the purpose. For many years now, this journal has offered a great opportunity for European scholars to communicate their findings to their peers and others. In its pages they have been able, for example, to discuss and compare the results when rules and regulations are applied to everyday situations in healthcare, and to propose methods whereby respect for the human rights and legitimate expectations of concerned parties can be ensured. I am honoured to be able to take part in the coming years in this important channel for diffusing the results of research on current and future issues relating to people's health.

\section{Demographic Challenges}

As noted, health law is an area that will face great challenges in coming years. This is particularly due to the demographic changes that many European countries are undergoing. These entail rapidly rising healthcare needs and costs, even as improvements in research, care, and services hold promise for meeting the challenges involved. One of the major achievements of this last century has been the dramatic increase in life expectancy. Due to rising living standards, 
better working conditions, and significant medical advances, people are living longer than ever before. Furthermore, their longevity is expected to increase still further. As a result, the average citizen in Europe is older than ever before.

Thus, one of the challenges we confront is the major demographic shift in Europe (and in many other parts of the world as well) towards an ageing society. This shift can be expected to bring major social, economic, and political consequences in its wake, with attendant risks for national healthcare systems. Responding to the ageing of the population is a policy priority for the EU, and the law is considered a key force for achieving the relevant structural changes. Among the legal areas being discussed, that relating to healthcare is central. The ageing of the population in the EU and worldwide, together with the fall in birth-rates, has prompted recurrent discussion about the urgent need for tailored care and greater cost-efficiency in the health sector. Spending on health is already substantial in the EU, accounting for 6-11 per cent of gross domestic product (GDP) in the member states. Among the member states in 2015, Germany had the highest ratio - $11.2 \%$ - and the costs in France and Sweden were nearly as high. ${ }^{1}$ Not surprisingly, in view of these considerations of both economy and quality, most of the Union's member states are very concerned with planning properly for the impact of demographic developments on future healthcare. Other legal areas affected by the demographic shift relate to the labour market, including various workplace-related issues, and to socialsecurity systems, including pensions and economic assistance. Increasingly, moreover, other challenges associated with demographic developments are being discussed as well. Housing for the elderly, for example, is a burning issue in Sweden and other countries, as are the legal, social, and ethical consequences of an increasingly globalised, digitalised, and privatised system of public healthcare.

\section{Digitalisation and Automated Decision-making}

Health law is also an area concerned with highly topical issues in a time of rapidly increasing digitalisation and automated decision-making in healthcare (AI), both in Europe and worldwide. The rapid developments in this area bring several critical issues to the fore, in connection for example with general data protection, intellectual property law, and the rights of patients. AI is expected to have a heavy impact on healthcare innovation and to influence all areas of healthcare. Developments in precision or personalised medicine, for example,

1 See https://ec.europa.eu/eurostat/statistics-explained/index.php/Healthcare_expenditure_ statistics. 
may revolutionalise healthcare in the future. Many hold high hopes for such progress on the EU level too, in connection among other things with reduced costs, improved standards of health, and easier access to healthcare.

The role of law in realising this scenario is crucial. Current laws must be reexamined in different ways, from the stage of innovation to that of application. Both the short- and the long-term consequences for the population must be addressed, among other things in connection with human rights, risks of discrimination, and concerns about vulnerability. The law can only be a factor in promoting a fair, responsible, and equitable implementation of AI in Europe if fundamental legal norms and well-tested legal principles are observed in healthcare. This includes the need for a proper interpretation of existing rules. Rules and regulations must be applied to everyday situations in healthcare in such a manner as to ensure respect for the legal rights and expectations of concerned parties, such as patients, medical staff, public-health authorities, financing institutions, and the like. If AI developments in healthcare are to have their desired effect, due consideration will need to be shown to the law, to the needs of patients, and to the fundamental rights of the individual, in accordance with national and European public values. This means that legal research on different matters relating to the AI trend will be central - from a global, national, community, and individual perspective.

\section{Research and Development}

Due account will also need to be taken of the ethical and legal issues arising in connection with AI and other advancements in healthcare. We need intensified medical research if we are to make progress in new medicines and alternative forms of treatment. The $\mathrm{EU}$ is providing substantial assistance for research in this and other fields. If Horizon 2020 was the largest ever researchand-innovation programme, with nearly 80 billion EUR of funding for 2014 to $2020,{ }^{2}$ Horizon 2030 will certainly not be smaller. The development of more advanced and personalised healthcare is important, but it also entails methods of medical research that often have legal implications. Such methods may involve, for example, the use of big data, with an increased collection and utilisation of digital health data. The use of such techniques is expected to be helpful among other things for treating neurodegenerative diseases like dementia. In Sweden, qualitative registries are one type of big data that is expected to prove particularly valuable for future health and welfare. These registries contain individualised data on patients' illnesses, medical interventions, and outcomes

2 See https://ec.europa.eu/programmes/horizon2020/en/what-horizon-2020. 
after treatment. They can be very useful in research aimed at finding future treatments. However, the use of such data can pose problems from the standpoint of patients' privacy. For example, the participation of patients is often needed if treatment for dementia is to be successful. This type of research raises some tricky legal questions in connection with the individual's right to integrity and privacy. Another method that may have ethical as well as legal implications is clinical research, because it directly involves a particular person or group of people; or it may involve the use of material from humans, such as tissue samples. Clinical trials and observation studies may entail the use of humans in various ways. In the case of clinical trials, the persons in question receive various forms of interventions (medicines, drugs, devices, etc.); in the case of observation studies, different types of observations are involved. Due to the information they provide about the benefits and risks of new drugs, interventions, living conditions, lifestyles, and the like, such studies can make a significant contribution to the care of future patients. In addition, however, due to the fact that the patients whose data is used in a medical study usually do not benefit from the study themselves, such research can pose issues relating to risk, privacy, and pain. Research of this kind reflects the need for a solid legal framework that can adapt to future needs in areas like digital privacy and intellectual property. Such a framework must include EU legislation and international treaties on the one hand, and legal provisions of a mostly national character on the other (as in some areas of health law, private law and administrative law).

European Conference on Health Law

Innovation in healthcare is also the topic of the 7 th European Conference on Health Law, to be held in Toulouse on 25-27 September 2019. The proceedings, organised by the European Association of Health Law (EAHL), will be headed by Anne-Marie Duguet. The theme of the conference, "New Challenges for Europe", reflects the times in which we live and work, where the role of the law in combating challenges is often critical for the outcome. This journal will arrange a panel, to be headed by Henriette Roscam Abbing, which will be presenting legal perspectives on some of the challenges mentioned in this editorial. I hope to see you at the conference.

\section{Titti Mattsson}

Professor, Faculty of Law, Lund University, Lund, Sweden; Editor, European Journal of Health Law 